\title{
Modeling and adaptive control for batch sterilization
}

\author{
Antonio A. Alonso ${ }^{1 *}$, Julio R. Banga ${ }^{2}$ and Ricardo Perez-Martin ${ }^{2}$ \\ ${ }^{1}$ Department of Chemical Engineering, University of Vigo, Vigo, Spain \\ ${ }^{2}$ Chemical and Food Engineering Group, Instituto de Investigaciones Marinas (CSIC). \\ Eduardo Cabello 6, 36208 Vigo, Spain \\ * Author to whom correspondence should be addressed. Fax: +34 86812 382. E-mail: \\ aalvarez@seinv.uvigo.es,
}

\begin{abstract}
This contribution addresses the control problem associated with tracking temperature profiles in batch sterilization processes. Dynamic variability of the plant and the unsteady state heat transfer to the product are shown to cause a significant performance degradation when simple PID-type controllers are employed to control the operation. This is especially true when operating at high temperature and low energy consumption. To improve performance, several adaptive control techniques such as selftuning regulator, stable adaptive control and adaptive IMC are compared on a simulation background, and their advantages and drawbacks discussed. In particular, it is shown that performance degradation often occurs at the early stages of recursive estimation, resulting in product overprocessing. To overcome such control degradation, a priori information derived from conservation principles of mass and energy, is incorporated into the control structure and combined with classical recursive identification techniques. This will avoid the appearance of high parameter uncertainty that otherwise would cause degradation of the closed loop response and/or instability. The complete scheme will be included into the IMC framework and their efficiency demonstrated on tracking sets of constant as well as variable (optimum) set point profiles for batch thermal processing.
\end{abstract}

Keywords: Adaptive control; Batch sterilization; IMC framework NOMENCLATURE 
$\mathrm{A}_{\mathrm{RT}}$ Effective retort area $\left(3.1 \mathrm{~m}^{2}\right)$

B Antoine's law parameter (4893.0 K)

$\mathrm{C}_{t}$ Characteristic parameter of valves (0.9)

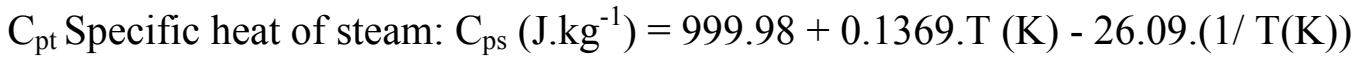

$\mathrm{C}_{\text {prod }}$ Specific heat of the product $\left(2.99 . \mathrm{kg}^{-1} \cdot \mathrm{K}^{-1}\right)$

$\mathrm{C}_{\mathrm{RT}}$ Specific heat of the retort body $\left(500 \mathrm{~J}-\mathrm{kg}^{-1} \cdot \mathrm{K}^{-1}\right)$

$\mathrm{C}_{\mathrm{vs}}$ Steam valve, characteristic parameter (12.6 gpm)

$\mathrm{C}_{\mathrm{vb}}$ Bleeder valve, characteristic parameter (1.4 gpm)

F Mass flow (kg.s $\left.{ }^{-1}\right)$

$\mathrm{F}_{\mathrm{o}}$ Microbiological lethality at the critical point (min)

h Enthalpy $\left({\mathrm{J} . \mathrm{kg}^{-1}}^{-1}\right)$

$\mathrm{h}_{\mathrm{c}}$ Convective heat transfer coefficient $\left(4.83 \mathrm{~J} . \mathrm{s}^{-1} \cdot \mathrm{m}^{-2} \cdot \mathrm{K}^{-1}\right)$

K Gain

$\bar{\kappa}$ Nominal gain

$\hat{\kappa}$ Gain estimated through recursive algorithms

m Accumulated mass $(\mathrm{kg})$

$\mathrm{M}_{\text {prod }}$ Total mass of the sterilized product $(100.0 \mathrm{~kg})$

$\mathrm{M}_{\mathrm{RT}}$ Effective retort mass $(345.0 \mathrm{~kg})$

$\mathrm{M}_{\mathrm{s}}$ Molecular weight of water

p Pressure (bar)

Q Heat (J)

R Constant of gases (8.34 J.mol.K ${ }^{-1}$ )

$\mathrm{T}$ Temperature $(\mathrm{K})$

$\mathrm{T}_{\text {ext }}$ External ambient temperature $(298 \mathrm{~K})$

$\mathrm{T}_{\text {prod }}$ Product temperature $(\mathrm{K})$

$\mathrm{T}_{\mathrm{s}}$ Sampling time (s)

$\mathrm{u}$ Valve position (from 0 to 1 )

$\mathrm{U}$ Internal energy $(\mathrm{J})$

$\mathrm{V}_{\mathrm{RT}}$ Retort volume $\left(0.3 \mathrm{~m}^{3}\right)$

W Mass flow through valves (kg. $\left.\mathrm{s}^{-1}\right)$

c Critical point

conv Convection term

prod Product

rad Radiation 
RT Interior of the retort

ref Reference state

s Steam

T Overall

Supersrcipts

eq Equilibrium state

i Input stream

Greek symbols

$\alpha$ Thermal diffusivity $\left(\mathrm{m}^{2} \cdot \mathrm{s}^{-1}\right)$

$\epsilon$ Bounds on the uncertainty

$\varepsilon$ Emissivity of the retort shell (0.94, dimensionless)

$\theta$ Stephan - Boltzmann constant $\left(5.68 .10 . \mathrm{s}^{-8} \mathrm{~J}_{\mathrm{s}} \mathrm{s}^{-1} \mathrm{~m}^{-2} \cdot \mathrm{K}^{-4}\right)$

$\lambda$ filter parameter (s)

${ }^{\wedge}$ Latent heat of water $\left(\mathrm{J} \cdot \mathrm{K}^{-1} \cdot \mathrm{kg}^{-1}\right)$

$v$ Thermal conductivity $\left(\mathrm{J} \cdot \mathrm{s}^{-1} \cdot \mathrm{m}^{-2} \cdot \mathrm{K}^{-1}\right)$

$\mathrm{T}$ Time constant (s)

$\bar{\tau}$ Nominal time constant (s)

$\hat{\tau}$ Time constant estimated through recursive algorithms (s)

\section{Introduction}

At present, an important number of industrial processes operate in batch mode. Greater flexibility (multiproduct capabilities), in response to fast changes in market conditions, high quality chemicals or small scale production of fine chemicals are the fundamental reasons that justify this mode of operation (Georgakis, 1990). Under these requirements, batch processing continues developing parallel to a growing market where demand requires a wider product spectrum (Shinskey, 1988).

Pharmaceuticals, polymers, biotechnology and the food industry constitute typical areas where discontinuous operation dominates over continuous. In the food industry, important operations such as drying, fermentation, evaporation and sterilization are usually carried out in batches (Bimbenet and Trystram, 1992). The latter, thermal 
sterilization in steam retorts is the process that prompted the present research. Two reasons justify this choice. In the first place, it is a high energy consuming operation frequently employed in almost all industries of the bioproducts sector. In the second place, the development of optimal control policies, together with the time varying nature of its dynamics and the uncertainty associated to the unsteady state heat transfer to the product, make this operation a challenging problem from a control point of view.

Sterilization is usually the limiting stage in the production chain. The objective consists of the inactivation by means of heat of possible spores, microorganisms or enzymes present in foodstuffs or bioproducts. This is carried out by steam flowing through a retort that contains the prepackaged product. Processing time and temperature are selected according to the mandatory degree of inactivation, as measured by the microbiological lethality. However, the thermal processing of the product also causes undesirable degradation of nutrients and sensorial quality parameters, such as color or texture (Lund, 1988). Banga et al. (1991), Banga et al. (1994) proposed different optimal temperature-time profiles for the thermal processing of conduction-heated foodstuffs, by solving the corresponding optimal control problem. They considered different objective functions involving nutrient retention, process time and energy consumption as well as constraints reflecting the required degree of inactivation. In this context, the present article refers to the implementation of those optimal profiles through the design of flexible and efficient controllers. By efficiency, we mean their capability to provide near optimum performance by avoiding offsets and/or prolonged overshoots that could result in overprocessing and serious quality degradation.

Additional design and operation constraints include:

1. The possibility of working under different operating conditions without the necessity of modifying the control structure and/or parameters.

2. To make use, as much as possible, of standard control equipment, mainly constituted by PID controllers.

From a control point of view, the plant, at least locally, can be considered first order. For that case, it is well known that high gain PI controllers are expected to perform well during the complete cycle (Mulvaney et al., 1990). This is true as long as the system is able to eliminate quickly the energy gained from the steam (Shinskey, 1988), which is not the case for most of the conditions met during the operation. In practice, such controllers are likely to lead to valve saturation and long overshoots which consequently 
will produce sensible overprocessing and energy losses. Other issues such as dynamic variability of the plant parameters (Lewin and Lavie, 1990) and the appearance of stability constraints, derived from the discrete implementation of the control algorithm, must also be considered.

Adaptive controllers could appear as the solution to the variability problem. Different types of adaptive control schemes were tested and compared in batch chemical reactors. Horak et al. (1982) and Kiparisides and Shah (1983) reported greater robustness and better performance than the traditional PID controllers. Takamatsu et al. (1985) combined, in what they called AIMC (adaptive internal model control), standard system identification techniques with internal model control design methodology (Garcia and Morari, 1982) for the control of batch polymerization reactors. Although such methodology proved better in performance than traditional PID controllers, the authors recognized the necessity of on-line tuning of filter parameters in order to improve the type of response. Adaptive techniques were also suggested by Mulvaney et al. (1990) for the case of batch sterilization although their performance, to the best of our knowledge, was never reported. Modeling and adaptive control for batch sterilization although their performance, to the best of our knowledge, was never reported.

In this paper we first evaluate some relevant adaptive control techniques employed to control batch systems, namely the selftuning regulator (STR) of Astrom and Wittenmark, stable adaptive controllers (Kiparissides and Shah, 1983), and the adaptive version of IMC developed by Takamatsu et al. (1985). Their performance will be compared with simple PID-type controllers and their advantages and drawbacks discussed. In particular, it will be shown that serious performance degradation often occurs during recursive estimation. This is due to the activation of input constraints and long excursions of the parameter estimates away from the current operation region. In order to avoid such undesired effects, the information provided by conservation principles of mass and energy will be brought into the control loop by open loop estimators. In this way, we extend the method adopted by Takamatsu et al. (1985) by combining parameter estimation from recursive least squares rules and open loop estimators derived from local linearization of the physical model of the plant. This will allow us to bound the allowable uncertainty and reject highly uncertain parameters obtained at the first stage of estimation. We note that parameter uncertainty between 
plant and model can result in a significant performance degradation, or even instability if time delays or hold elements are present in the loop. With this information, filter parameters can be tuned, even on-line, to improve closed loop performance while avoiding possible instabilities inherent to the discrete controller implementation.

The paper is organized as follows. The sterilization process in batch units as well as the difficulties related to the operation will be described in Section 2. In Section 3 we compare, through simulations, the efficiency and performance of classical constant parameter PI controllers and some standard adaptation techniques such as the STR and stable adaptive controllers (Kiparisides and Shah, 1983). The proposed control scheme, including the different adaptation methods, switching rules and filter tuning laws is developed in Section 4. In that section, the performance of this technique in carrying out constant as well as variable temperature set point profiles is demonstrated through simulation.

2. Thermal processing in batch units: model description

Here, we briefly describe the operation under study. For a more detailed description of a traditional sterilization process the reader is referred to Lopez (1987). The mathematical model, derived from mass and energy balance equations, is presented in Appendix 5.1. A first order linear approximation of this model will be employed to design open loop adaptation schemes that will be included in the control loop.

\subsection{Sterilization in batch units}

Sterilization is carded out in batch steam retorts as that depicted in Fig. 1. A typical operation cycle involves several stages, namely venting, heating and cooling. In the first stage (venting), the air present in the retort is eliminated from the vessel with saturated steam. This avoids the presence of steam-air mixtures that would prevent the sterilization standards to be met during the next cycle (heating). At this stage bleeder and drain valves are fully open. When the pressure in the retort matches that corresponding to saturated steam at that temperature, air is considered absent from the retort and the heating cycle starts. Along this stage, the product is usually kept at a working constant temperature for a certain time, prescribed by the desired 
microbiological lethality F 0 . This variable, which describes the degree of microbiological inactivation, is estimated at the critical point (the coldest point of the product during the heating stage) according to the following equation:

$$
F_{o}=\int_{0}^{1} 10 \frac{T_{c}(t)-T_{r e f}^{k}}{D} d t
$$

where $\mathrm{D}$ represents a kinetic parameter describing the thermal destruction of a certain spore and $T_{r e f}^{k}$ its reference temperature. $T_{c}$ corresponds to the temperature at the critical point. Due to the exponential-type relationship between temperature and lethality, small disturbances or prolonged overshoots in temperature could result in serious under- or overprocessing of the product. The former implies health unsafety and therefore waste or reprocessing of the batch while the latter usually implies high quality degradation. Consequently, a fine temperature control is required during this cycle. To illustrate the effect of an offset in the retort temperature on the microbiological lethality (as defined in (1)), let us consider a convective heated product. The increase in lethality due to a positive deviation on the retort temperature of $\Delta T_{R}$ will be of the order:

$\frac{\Delta F_{o}}{F_{o}\left(T_{R}\right)}=10 \frac{\Delta T_{R}}{D}-1$

For a linear temperature-time profile $T_{R}=a t+b$, the relative increase in lethality takes the form:

$$
\frac{\Delta F_{o}}{F_{o}\left(T_{R}\right)}=10 \frac{\Delta b}{D}-1
$$

Assuming some standard values for the kinetic parameters $\left(\mathrm{D}=10^{\circ} \mathrm{C}\right.$ at $\mathrm{T}_{\mathrm{ref}}=121.11^{\circ} \mathrm{C}$, and $1{ }^{\circ} \mathrm{C}$ deviation from the set point), the relative increase in lethality for both cases is about $25 \%$. This amount, negligible at the beginning of the heating cycle, can lead to a severe overprocessing as time progresses. Consequently, overshoots and offsets must be avoided especially when approaching to the prescribed lethality.

Once the heating period concludes, the product is cooled with water to room temperature. At this stage, air is injected into the vessel to avert sudden pressure drops that could result in the bursting of product containers. Pressure control during this stage is especially important for glass containers or conduction heated-type products where 
the existence of sharp temperature gradients between the inside and the outside of the product induces high differential pressures (Alonso et al., 1993).

2.2. Dynamic variability of the plant. A linear approximation to its dynamic behavior

The model equations, based on mass and energy balances are presented in Appendix 5.2. Formally, the heating stage can be represented as follows:

$$
\Omega \dot{T}_{R T}=W_{s}\left(T_{R T}\right) u_{s}-W_{b}\left(T_{R T}\right) u_{b}-\frac{\dot{Q}_{T}+\dot{Q}_{p r o d}}{\Lambda}
$$

where $\Lambda$ represents the latent heat, assumed constant, and $\Omega$ takes into account nonlinear terms of the form:

$$
\Omega\left(T_{R T}\right)=\left(\frac{p^{e q} V_{R T}}{r_{8} T_{R T}^{3}}\left(B-T_{R T}\right)+\frac{m_{R T} C_{R T}}{\Lambda}\right)
$$

From (4) we construct a linear approximation of the plant by expanding the model in Taylor series around the current operation point $-\overline{\mathrm{T}}_{R T}, \bar{u}_{s}, \bar{u}_{b}$-considered as the steady state reached by fixing steam and bleeder valves and letting $\dot{Q}_{\text {prod }}=0$. This results in a first order differential equation of the form:

$$
\tau \Delta \dot{T}_{R T}+\Delta T_{R T}=K_{s} \Delta u_{s}-K_{b} \Delta u_{b}-g\left(\Delta T_{R T}\right)
$$

where $\Delta$ represents deviation from the current operation point. A detailed derivation of (6) is presented in Appendix 5.2. The function $\mathrm{g}\left(\Delta \mathrm{T}_{\mathrm{RT}}\right)((\mathrm{A} 17))$ includes the effect of unsteady state heat transfer to the product (in this contribution, considered of conductive nature). This term will he omitted in the development of the control structure since no feed-forward schemes are considered. Their time and space distributed nature (heat is mainly absorbed by conduction) would result in highly computational expensive estimators. However, the effect of $\mathrm{g}\left(\Delta \mathrm{T}_{\mathrm{RT}}\right)$ on the dynamic is taken into account by assuming that the parameters $\tau, \mathrm{K}_{\mathrm{s}}$ and $\mathrm{K}_{\mathrm{b}}$, state dependent, are subject to multiplicative uncertainties $\alpha_{\mathrm{t}}$ and $\alpha_{\mathrm{k}}$, respectively.

The variability of gains and time constant with the operation conditions is a direct consequence of the batch nature of the process, the non-stationary heat transfer and the interaction between bleeder and steam valves. In this way, increasing the size of the valves would increase the operability region (understood as the region where changes in 
the input affect the output) and attenuate variability, but at the expenses of narrowing the range of available working temperatures and, consequently, weakening the flexibility of the unit (to implement time varying temperature profiles, for instance). The effect of steam valve positions on gain and stationary temperature for our case study is presented in Fig. 2. The operability region, defined as the region where changes in the input affect the output, is limited to steam valve positions $\left(\mathrm{u}_{\mathrm{s}}\right)$ smaller than 0.2 for all the positions of the bleeder $\left(\mathrm{u}_{\mathrm{b}}\right)$. There, gain suffers abrupt variations spanning the set of available operation temperatures (as it can be seen in Fig. 2 (b), from $100^{\circ} \mathrm{C}$ to $140^{\circ} \mathrm{C}$ ). We note that the possibility of working at low bleeder openings (shorter operability regions) is greatly desirable from an economic point of view since it would lead to considerable energy savings.

To illustrate the effect of such variability on controller performance, a constant parameter PI controller designed by IMC (Internal Model Control) (Rivera et al., 1986), and the equivalent one with variable parameters, adapted according to (A14)(A15), are compared in tracking different temperature transitions at low temperature (bleeder fully open) and high temperature operations (low bleeder opening). For the sake of completeness, the controller structure is summarized next.

\subsubsection{Controller transfer function}

$$
c(s)=\frac{q}{1-\bar{p} q}
$$

where

$$
q(s)=\frac{1}{\bar{p}(s)} f(s)
$$

$\mathrm{f}(\mathrm{s})$ is a low pass filter of the form:

$$
f(s)=\frac{1}{\lambda s+1}
$$

and the plant is represented in Laplace Domain as:

$$
\bar{p}(s)=\frac{K_{s}}{\tau s+1}
$$


Substituting (10) and (9) in (7), the corresponding PI controller (in velocity form) becomes:

$U^{k}=u^{k-1}+\frac{\tau}{K_{s} \lambda}\left(e^{k}-e^{k-1}\right)+\frac{1}{K_{s} \lambda} e^{k} T_{s}$

The responses obtained by constant and variable parameters PI controllers are represented in Figs 3(a) and (b). As it can be seen from Fig. 3(a), the responses obtained for a temperature transition from $103^{\circ} \mathrm{C}$ to $110^{\circ} \mathrm{C}$ with $\mathrm{u}_{\mathrm{b}}=1$ show little or negligible differences in performance. Unfortunately, such controlled behaviour can no longer be obtained with simple PI controllers when higher operation temperatures are required. This case is illustrated in Fig. 3(b) where the desired temperature profile is a step from $103^{\circ} \mathrm{C}$ to $130^{\circ} \mathrm{C}$ for a bleeder position $\mathrm{u}_{\mathrm{b}}=0.25$. In this experiment, the application of constant parameter PI controllers, designed by considering the nominal plant at fixed operation temperatures (CIMC1 and CIMC2) resulted in excessive overshoots. In $\mathrm{CIMC1}$, the parameters of the IMC controller $\mathrm{K}_{\mathrm{s}}$ and $\mathrm{T}$ have been derived from (A14) and (A15), respectively, at $\bar{T}_{R T}=130^{\circ} \mathrm{C}, \bar{u}_{b}=0.25$ and $\bar{u}_{s}=0.045$ which corresponds to the desired operation point. In CIMC2 the parameters have been chosen at $\bar{T}_{R T}=132^{\circ} \mathrm{C}$, $\bar{u}_{b}=1$ and $\bar{u}_{s}=0.15$. These tests suggest the application of adaptive control strategies, capable of taking into account the dynamic variability. This is the objective that will be pursued in the following section.

3. Testing of standard adaptive control techniques

Variation of plant parameters in (10) can be captured by either using standard adaptive techniques or by scheduling the parameters of the controller through (A14) (A15) (A16) as it was shown in the Section 2.2. Here, we first implement and compare some standard adaptive control techniques, including adaptive IMC controllers, for the heating stage of a batch sterilization process. The main issues associated with these techniques will be outlined and will justify a hybrid adaptive approach that combines information based on conservation principles with that obtained through on-line inputoutput recursive identification techniques.

\subsection{Adaptive control algorithms}


For the sake of completeness, we summarize next the algorithms employed:

\subsubsection{Model of the plant}

The input-output behavior of the plant is represented in a discrete version as follows:

$y(t)=a\left(z^{-1}\right) y(t)+b\left(z^{-1}\right) u^{\prime}+\epsilon(t)$

where:

$\mathrm{a}\left(\mathrm{z}^{-1}\right)=\mathrm{a}_{\mathrm{o}}+\mathrm{a}_{1} \mathrm{z}^{-1}+\ldots+\mathrm{a}_{\mathrm{m}} \mathrm{z}^{-\mathrm{m}}$

$\mathrm{b}\left(\mathrm{z}^{-1}\right)=\mathrm{b}_{\mathrm{o}}+\mathrm{b}_{1} \mathrm{z}^{-1}+\ldots+\mathrm{b}_{1} \mathrm{z}^{-1}$

$\mathrm{u}^{\prime}=\left(1-\mathrm{z}^{-1}\right) \mathrm{u}$

$\mathrm{z}^{-1}$ represents, as usual, the backwards difference operator, $\epsilon(\mathrm{t})$ is a moving average error term, uncorrelated with the input and output variables, $y$ and $u$ represent the output and input deviation with respect to some steady state point, respectively.

\subsubsection{Parameter estimation}

The parameters of the model will be estimated by standard recursive least squares techniques. Next, we state the relations employed. Rigorous derivations can be found elsewhere (Astrom and Wittenmark, 1984):

$$
\begin{aligned}
\theta(\mathrm{t}) & =\theta(\mathrm{t}-1)+\mathrm{K}(\mathrm{t})\left(\mathrm{y}(\mathrm{t})-\varphi(\mathrm{t}-1)^{\mathrm{T}} \theta(\mathrm{t}-1)\right) \\
K(t) & =\frac{P(t-1) \varphi(t-1)}{k+\varphi(t-1)^{T} P(t-1) \varphi(t-1)} \\
P(t) & =\frac{1}{k}\left[P(t-1)-\frac{P(t-1) \varphi(t-1) \varphi(t-1)^{T} P(t-1)}{k+\varphi(t-1)^{T} P(t-1) \varphi(t-1)}\right]
\end{aligned}
$$

where $\theta$ and $\varphi$ are, respectively:

$\theta^{\mathrm{T}}(\mathrm{t})=\left(\mathrm{a}_{0}, \ldots, \mathrm{a}_{\mathrm{m}}, \mathrm{b}_{0}, \ldots, \mathrm{b}_{\mathrm{t}}\right)^{\mathrm{T}}$

$\varphi^{\mathrm{T}}(\mathrm{t})=(\mathrm{y}(\mathrm{t}), \ldots, \mathrm{y}(\mathrm{t}-\mathrm{m}), \mathrm{u}(\mathrm{t}), \ldots, \mathrm{u}(\mathrm{t}-\mathrm{l}))^{\mathrm{T}}$

$\mathrm{k}(0<\mathrm{k}<\mathrm{l})$ represents the forgetting factor and $\mathrm{p}^{-1}$ is some positive definite matrix called the covariance matrix. It can he interpreted as a measure of the uncertainty of the parameters in which case it is defined as $\mathrm{P}^{-1}(\mathrm{t})=\mathrm{E}\left[\theta(\mathrm{t}) \theta^{\mathrm{T}}(\mathrm{t})\right] \cdot \theta(\mathrm{t})$ represents a deviation with respect to the "true" plant parameters. The following adaptive laws are considered: 
The resulting minimum variance control law is given by:

$u^{\prime}=\frac{a\left(z^{-1}\right)}{b\left(z^{-1}\right)} y(t)$

3.1.4. Stable adaptive control (Goodwin et al., 1980)

The corresponding adaptive control law is in this case of the form:

$u(t)=\frac{1}{b_{0}}\left(y_{s p}(t)-\varphi^{T}(t) \theta(t)\right)$

where $\mathrm{y}_{\mathrm{sp}}(\mathrm{t})$ represents the desired output at time $\mathrm{t}$ and $b_{0} \neq 0$

\subsection{Performance of the adaptive controllers}

The controllers ((17) and (18)) were tested in controlling temperature during the heating stage of a batch sterilization cycle. The plant is modelled by a discrete input-output difference equation of the form of (12) with polynomials $\mathrm{a}\left(\mathrm{z}^{-1}\right)$ and $\mathrm{b}\left(\mathrm{z}^{-1}\right)$ of degrees $\mathrm{m}=$ $1, \mathrm{~m}=2$ and $\mathrm{l}=0,1=1$, respectively. Parameters were estimated recursively by (14)(15)(16). Persistent excitation was insured by introducing a PRBS (pseudo random binary signal) at the input $u$. The performance of the stable adaptive control (SAC) and the evolution of the parameters is illustrated in Fig. 4 for a sequence of ramptype temperature profiles and a sampling time of $2 \mathrm{~s}$. Steps were substituted by ramps in order to avoid excessive movements of the manipulated variable that could result in valve saturation. As it is seen in Fig. 4(a), the response exhibits a persistent offset that will result in overprocessing of the product. The provision for incremental control by switching off the adaptation gain when the error is small (see, for instance Seborg et al., 1986) did not improved the response either. Different simulation experiments showed that offsets and valve saturation became more pronounced as the slope of the set point profiles and the sampling time increased. Integral terms made the offset disappear although at the expenses of unacceptable oscillations. The STR ((17)) was tested on tracking step changes in the set point from $125^{\circ} \mathrm{C}$ to $130^{\circ} \mathrm{C}$. Larger transitions resulted in too oscillatory behavior and valve saturation. In Fig. 5(a) and (b), the evolution of 
temperature is represented for first and second order models, respectively. Oscillations became more pronounced as the sampling time increased.

After several trials, the forgetting factor was tuned to a value of $k=0.3$. Higher values promoted long excursions of the parameters far from the nominal ones with smaller parameter convergence rates. As a result, performance degradation or instability occurred. Such small forgetting factor reflects the high variability of the plant parameters (especially when operation is under low bleeder openings). Information must be discharged quickly in order to match the nominal plant parameters. Some important observations can be extracted out of these tests:

- Performance is highly dependent on the choice of the algorithm parameters. In many situations, bad initial parameter estimates and forgetting factors resulted in long excursions of the estimates in the parameter space. The ultimate consequence of this behavior was a significant performance degradation and instability.

- Due to the batch nature of the process, it is not unusual for some of the trajectories to induce large input movements that result in valve saturation. At that point, persistent excitation fails and parameter drift occurs.

\subsection{Adaptive IMC}

Internal model control can be considered as a methodological environment to design stable control structures according to $\mathrm{H}_{2}$ or $\mathrm{H}_{\infty}$ optimallity criterion. It provides practical guidelines to derive robust controllers with close to optimum performance behavior as well as a simple way to handle uncertainty. Among others advantages, we draw attention its simplicity and capability to handle input saturation constraints and output responses, by properly selecting the filter parameters.

The combination of recursive estimation and IMC was adopted by Takamatsu et al. (1985) for the control of batch polymerization reactors and it will be analyzed here for our case study through simulation. A schematic of the control structure is presented in Fig. 6 where the estimator consists of $(14)(15)(16)$ and the IMC controller is derived from $(7)(8)(9)(10)(11)$. Gain and time constant are computed from the discrete model of (12) through the following equivalences $\left(\mathrm{u}^{\prime}=\mathrm{u}, \mathrm{m}=1\right.$ with $\mathrm{a}_{0}=0$ and $\left.\mathrm{l}=0\right)$ : 
$a_{1}=\exp \left(-\frac{T_{s}}{\hat{T}}\right)$

$b_{1}=\hat{K}_{s}\left(1-\exp \left(-\frac{T_{s}}{\hat{T}}\right)\right)$

where the symbol ${ }^{\wedge}{ }^{\prime}$ stands for the estimated values of gain and time constant. Similar relationships can be derived for higher order models. Figures 7 and 8 show the response obtained for several step changes in the set point as well as the evolution of the recursively estimated parameters (REP) and their corresponding nominal values (MEP), computed from (A14) and (A15). A disturbance in the bleeder valve position from 0.25 to 0.5 , was introduced at $800 \mathrm{~s}$. It is noted that parameter convergence is finally achieved after each step in the set point. However, the earlier estimates stay far away from the nominal ones. This results in valve saturation and consequently significant performance degradation compared with the response obtained by updating the parameters through open loop estimators (e.g. compare with Fig. 3(b)). We also note the possibility of sign change in parameters and of parameter burst (see Fig. 8) as estimation proceeds thus causing poor tracking and instability. These effects become more apparent as the sampling time increases. The response obtained for a sampling time of $10 \mathrm{~s}$ is presented in Fig. 9.

\section{Adaptive imc controller: a hybrid approach}

From the comparison made on the last section, one almost obvious conclusion can be drawn: if an accurate dynamic model of the plant is available, open loop estimators represented by (A14)(A15)(A16) will give a much better controller performance than any recursive estimation technique. This has been already pointed out by Lewin and Lavie in their work on the design of exothermic batch chemical reactors (Lewin and Lavie, 1990). However, uncertainty is always present due to unexpected disturbances or to uncertainty of the physical parameters describing the plant. Into the first category, we can consider different product loads processed from batch to batch or unmeasured boiler temperature changes due to an extra demand. Heat transfer coefficients or changes in valve dynamic parameters belong to the second category. Such uncertainties often translate into poor tracking capabilities or even instability in digital control applications. 
In the present approach, we recognize the existence of inherent uncertainty in the model parameters estimated at the model validation stage. Therefore, we propose a combination of recursive estimation (RE) and open loop estimation (OE) according to the current observed uncertainty in the RE parameters with respect to their nominal equivalent (those computed through $\mathrm{OE}$ ). This idea is formalized as follows:

$K=\bar{K} \cdot\left(1+\alpha_{k}\right)\left|\alpha_{k}\right| \leq \epsilon_{k} \leq 1$

$\mathrm{T}=\overline{\mathrm{T}} \cdot(1+\alpha \mathrm{T})\left|\alpha_{t}\right| \leq \in \mathrm{T} \leq 1$

$\overline{\mathrm{T}}$ and $\bar{K}$ correspond to (A14)) and (AI5), respectively and $\epsilon_{\mathrm{k}}, \epsilon \mathrm{T}$ are bounds on the uncertainty, computed at the model validation stage. (20) suggests the following switching between RE and OE.

\subsubsection{Switching algorithm}

IF $\frac{\hat{\tau}}{\bar{\tau}}-1 \leq \varepsilon \tau$ AND $\frac{\hat{K}}{\bar{K}}-1 \leq \varepsilon_{k}$ THEN $\mathrm{K}=\hat{K}, \tau=\hat{\tau}$ ELSE $\mathrm{K}=\bar{K}, \tau=\bar{\tau}$

$\hat{K}$ and $\hat{\tau}$ correspond to the values obtained from RE. The approach pursued here is inspired in the work of Takamatsu et al. (1985) and Lewin and Lavie (1990) on adaptive IMC. It extends their work to address stability issues derived from high plant uncertainty and performance degradation due to parameter drift. Since the system is first order and gain does not change sign, it is obvious that the closed loop will be stable for all positive filter constants. However, in the discrete case, large uncertainties may induce instability for a fixed filter constant. That issue will be addressed in the next section.

\subsection{Stability conditions of model based IMC controllers}

In this section, we derive relations between filter time constant and uncertainties in the plant parameters. These will be included in the control structure to preserve closed loop stability. Essentially, we follow the same line of arguments as those employed by Rotstein and Lewin (1991) to relate the minimum allowed filter constant to a function of the sampling time and the uncertainty of both plant gain and time constant. Uncertainties are computed by solving (20) for $\alpha_{\mathrm{k}}$ and $\alpha_{\mathrm{T}}$ where $\mathrm{K}$ and $\mathrm{T}$ take the values 
dictated by the algorithm of $(21)(22)$. In the $\mathrm{z}$ domain, the discrete PI controller ((11)) becomes:

$$
\begin{aligned}
& c(z)=\frac{\hat{\tau}}{\hat{K} \lambda y} \frac{z-y}{z-1} \\
& y=\frac{\bar{\tau}}{\bar{\tau}+T_{s}}
\end{aligned}
$$

For the DDC (direct digital control) structure, the plant of (10), with a zero order hold, takes the form:

$$
(H p)(z)=\bar{K}\left(1+\alpha_{k}\right) \frac{1-b}{z-b} \text { and } b=\exp \left(\frac{-T_{s}}{\bar{\tau}\left(1+\alpha_{\tau}\right.}\right)
$$

The marginal conditions for stability are obtained from the closed loop plant as:

$$
F(z)=(H \bar{p})(z) C(z 0)=-1
$$

From the Nyquist plot it can he seen that the intersection with the negative real axis occurs at $\omega=\frac{\pi}{T_{s}}$. This leads to the following lower bound for the filter constant which preserves stability:

$$
\lambda>\frac{(1-b)}{(1+b)}\left(\bar{\tau}+\frac{T_{s}}{2}\right)\left(1+\alpha_{k}\right)
$$

The same technique is employed for a second order filter of the form:

$$
f(s)=\frac{2 \lambda s+1}{(\lambda s+1)^{2}}
$$

Substitution of this filter and the plant into (7) results in a PII ${ }^{2}$ controller which in velocity form becomes:

$u^{k}=u^{k-1}+\frac{2 \bar{\tau}}{\bar{K} \lambda}\left(e^{k}-e^{k-1}\right)+\left(\frac{\bar{\tau}}{\bar{K} \lambda^{2}}+\frac{2}{\bar{K} \lambda}\right) e^{k} T_{s}+\frac{T_{s}^{2}}{\bar{K} \lambda^{2}} \sum_{j=0}^{k} e^{1}$

In the $\mathrm{z}$ domain, (28) results in the form:

$$
c(z)=\frac{\left(z-r_{1}\right)\left(z-r_{2}\right)}{(z-1)^{2}}
$$


with:

$$
\begin{aligned}
& r_{1}=\frac{\bar{\tau}}{\bar{\tau}+T_{s}} \\
& r_{2}=\frac{2 \lambda}{2 \lambda+T_{s}}
\end{aligned}
$$

Applying the condition of (25) with $\mathrm{c}(\mathrm{z})$ as in (29) and (Hp)(z) as in (24), and noting that the intersection occurs at $\omega=\frac{\pi}{T_{s}}$, a stable filter time constant must be bounded as:

$$
\lambda>\frac{\phi-1}{2-\phi} \frac{T_{s}}{2}
$$

with:

$\phi=\frac{4}{\bar{K}\left(1+\alpha_{k}\right)} \frac{(1+b)}{(1-b)} \frac{1}{(1+y)}$

and y as in (23). In order to assure stability, (26) or (30) should be employed at the control design level, based on the expected uncertainty and sampling time.

Alternatively, they could also be included into the control structure and checked at every sampling time after (20).

\subsection{Performance of the hybrid controller}

In this section, we present the results on the performance exhibited by the hybrid adaptive IMC controller in tracking step and ramp type set point profiles. In Fig. 10 we represent the temperature response and parameter evolution for a situation in which $\epsilon_{\mathrm{T}}=$ $\epsilon_{\mathrm{k}}=0.5$. As is expected, the switching algorithm prevents sign change in the plant parameters, spikes and parameter drifts and therefore improves performance considerably. This is observed from the comparison with Figs 8 or 9. This control structure results especially attractive when carrying out variable (time dependent) temperature profiles. In this case, a linearization around a single operating point is not possible due to the unsteady nature of the set point trajectory. This type of profile, represented in terms of ramp functions, spans a wide range of temperatures, so a constant parameter controller would lead to responses that are extremely fast or slow, depending on the states region and the model parameters selected. To carry out free- 
offset tracking, a filter of the form of (27) was employed (Deshpande, 1992), resulting in a $\mathrm{PII}^{2}$ controller of the form (28).

The performance of the hybrid IMC controller in carrying out variable temperature set point profiles was tested on simulation. To that purpose, a number of RO-100 metal cans (cylindrical geometry of radius $3.26 \mathrm{~cm}$ and height $3.0 \mathrm{~cm}$ ) with a total volume of $0.1 \mathrm{~m}^{3}$, was considered inside the retort, being the diffusivity of the product $\alpha=$ $1.544 .10^{-7} \mathrm{~m}^{2} \mathrm{~s}^{-1}$. After venting, the temperature in the retort was $103^{\circ} \mathrm{C}$. The maximum model uncertainty employed in (20) was $\epsilon_{\mathrm{k}}=\epsilon_{\mathrm{T}}=0.95$. These values were obtained from previous simulations where $\mathrm{g}\left(\Delta \mathrm{T}_{\mathrm{RT}}\right)$ was estimated from the integration of the Fourier equation for the product (see the Appendix 5.1). The required temperature profile corresponds to that which minimizes process time for a certain microbiological lethality (see Banga et al. (1991)). The performance of the hybrid strategy is shown in Figs 11 and 12. Figure 11(a) represents the evolution of temperature in the retort and at the center point of each product container as well as the movements of the manipulated variable. The evolution of the gain and the time constant is presented in Fig. 11(b).

It is noted that some mismatch occurs between the actual temperature response and the set point at the end of the cycle (descendent ramps). This reflects the appearance of physical constraints affecting the energy transfer with the environment. The controller reacts to the set point change by closing the steam valve. However, the energy dissipation rate required by this change is greater than that produced by losses through the bleeder and by radiation and convection. Consequently, the retort temperature remains over the specified trajectory. In practice, the cooling cycle should start at this point, by introducing cooling water. Air must also be open in order to compensate for sudden pressure drops that could result in bursting of the containers. A detailed study of the design of IMC controllers for pressure 5, Conclusions control during the cooling stage can found in Alonso et al. (1993).

During the cooling stage water is flowing into the retort at a constant rate of $F_{w}=0.5$ $\operatorname{ldotmin}^{-1}$ while a PI controller acts on the air flow to compensate for pressure changes. After a certain period (when pressure increases over a certain safety value) the control of pressure is transferred to the drain valve which at the same time keeps a constant liquid level into the retort. The evolution of temperature and pressure for the complete 
cycle is presented in Fig. 12. Cooling starts after $3500 \mathrm{~s}$ to avoid the mismatch between the current temperature and the set point. As is seen in Fig. 12(a), the combination of heating and cooling stages results in a perfect ramp tracking of the retort temperature. Figure 12(b) and (c) represent the evolution of pressure and liquid level as well as the movements of the manipulated variables (air flow and drain opening). Although the liquid level loop shows a slow dynamic, a stationary state is reached at the safety level of 1301 (the available volume is 2501 for the loaded retort).

\section{Conclusions}

This contribution describes the most relevant issues associated to the design of control structures for batch processes. Adaptation is justified on the need to account for the dynamic variability of the plant, valve interactions and unmodelled dynamics such as the unsteady state type of heat transfer to the product. However, estimation based only on input--output information can lead to unacceptable performance due, for instance, to parameter drift or valve saturation. Therefore, adaptation must be complemented with a priori information derived from first principles models. Such information can be handled on the basis of uncertainty estimation through simple rules of the form of (20) included in the control structure.

These ideas have been illustrated by simulation for the control of temperature in batch sterilization. Hybrid adaptive controllers designed on a IMC framework proved to perform efficiently in tracking constant as well as variable time-temperature profiles. This approach resulted specially attractive at high temperatures and low energy consumptions rates as well as in carrying out time varying temperature profiles, where a linear representation of the plant around operating points is not possible.

Acknowledgements

This work was financially supported by a research grant from the Comision Interministerial de Ciencia y Tecnologia, CICYT project ALI91-0712, Spain. We also wish to thank the anonymous reviewers for their valuable comments. 
Alonso, A. A., Desarrollo de estrategias de control avanzado para el procesamiento termico de alimentos en unidades discontinuas. Ph.D. Thesis, Dept. Ingenieria Quimica, Universidad de Santiago de Compostela, Spain (1993).

Alonso, A. A., Banga, J. R. and Perez-Martin, R. I. (1993) A new strategy for the control of pressure during the cooling stage of the sterilization process in steam retort: Part I. A preliminary study. Trans. IChemE C. 71(9), 197

Astrom, K. J. and Wittenmark, B., Computer Controlled Systems. Prentice Hall, Englewood Cliffs, N.J. (1984).

Banga, J. R., Perez-Martin, R. I., Gallardo, J. M. and Casares, J. J. (1991) Optimization of the thermal processing of conduction-heated canned foods: Study of several objective functions. J. Food Engng. 14, 25

Banga, J. R., Alonso, A. A. and Perez-Martin, R. I. (1993) Mathematical modeling and simulation of the thermal processing of anisotropic and non-homogeneous conduction-heated canned foods: Application to canned tuna. J. Food Engng 18(4), 389

Banga, J. R., Alonso, A. A., Perez-Martin, R. I. and Singh, R. P. (1994) Optimal control of heat and mass transfer in food and bioproducts processing. Comput. Chem. Engng 18S, 699

Bimbenet, J. J. and Trystram, G. (1992) Process control in food industry. Trans. IChemE C 70(9), 115

Bhowmik, S. R., Vichnevetsky, R. and Hayakawa, K. (1985) Mathematical model to estimate steam consumption in vertical still retorts for thermal processing of canned foods. Lebensm. Wiss. Und Technol. 18(1), 15

Clarke, D. W. and Gawthrop, P. J. (1975) Self-tuning controller. Proc. IEE 122, 929

Deshpande, P. B. (1992) On-line quality control with PID-type controllers. Chem. Engng Progress 88(5), 71

Garcia, C. E. and Morari, M. (1982) Internal Model Control 01. A unifying review and some new results. Ind. Chem. Engng Proc. Dev. 21, 308.

Georgakis, C., Opportunities and challenges of the optimization and control of batch processes, Generic Control for Batch Manufacturing. Purdue Research Foundation, Indiana (1990).

Goodwin, G. C., Ramadge, P. J. and Caines, P. E. (1980) Discrete-time multivariable adaptive control. IEEE Trans Aut. Control AC-25, 449 
Horak, J. E, Jiracek, C. and Jezova, L. (1982) Adaptive temperature control in chemical reactors. A simplified method maximizing productivity of a batch reactor. Coll. Czech. Chem. Comm. 47, 251

Kiparisides, C. and Shah, S. L. (1983) Self-tuning and stable adaptive control of a batch polymerization reactor. Automatica 29(3), 225

Lewin, D. R. and Lavie, R. (1990) Designing and implementing trajectories in an exothermic batch chemical reactor. Ind. Chem. Engng Proc. Dev. 29, 89

Lopez, A., A complete course in canning and related processes, Vol. I. The Canning Trade Inc., Baltimore (1987).

Lund, D. B., Effects of heat processing on nutrients. In Nutritional evaluation of food processing (Edited by E. Karmas and R. S. Harris). AVI Publishing Co., New York (1988).

Morari, M. and Zafiriou, E., Robust Process Control. Prentice Hall Inc., Englewood Cliffs, N.J. (1989).

Mulvaney, S. J., Rizvi, S. S. H. and Johnson, C. R. (1990) Dynamic modeling and computer control of a retort for thermal processing. J. Food Engng 11, 273

Rivera, D. E., Morari, M. and Skogestad, S. (1986) Internal Model Control-4. PID controller design. Ind. Chem. Engng Proc. Dev. 25, 252

Rotstein, G. E. and Lewin, R. (1991) Simple PI and PID tuning for open-loop unstable systems. Ind. Chem. Engng Proc. Dev. 30, 1864

Seborg, D. E., Edgar, T. E and Shah, S. L. (1986) Adaptive control strategies for process control: A survey. AIChE J. 32(6), 881

Shinskey, E G., Process Control Systems: Application, Design and Tuning. McCrawHill Inc., New York (1988).

Smith, C. A. and Corripio, A. B., Principles and practice of automatic process control. John Wiley, New York (1985).

Takamatsu, T., Shipya, S. and Okada, Y., Adaptive Internal Model Control and its application to a batch polymerization reactor, IFAC Conference on Adaptive Control of Chemical Processes. Frankfurt, Germany (1985).

Appendix

5.1. Model of a batch sterilization process 
A dynamic lumped parameter model, based on mass and energy balance equations, was presented by Alonso (1993). For the sake of completeness we summarize the equations employed. The different variables and parameters employed are summarized in the Nomenclature. The model is built assuming the steam, air and their mixtures (Bhowmik et al., 1985; Mulvaney et al., 1990) are ideal. The system, excluding the product, is assumed to be homogeneously heated. As reported by Alonso (1993), this is true for temperatures above the boiling point of water at atmospheric pressure. Other assumptions include saturation of the steam for most part of the heating cycle and absence of liquid water in the vessel (condensate is continuously drawn off the retort). Under these conditions, the mass and energy balances become:

Mass balance:

$\dot{m}_{s}=F_{s}-x_{s} F_{b^{\prime}}^{s}$

Energy balance:

$\dot{U}_{s}=h_{s}^{i} F_{s}-h_{s} x_{s} F_{b}^{s}-\dot{Q}_{T}-\dot{Q}_{R T}$

The internal energy $\left(U_{s}\right)$ is represented as a function of the enthalpy:

$\mathrm{U}_{\mathrm{s}}=\mathrm{m}_{\mathrm{s}}\left(\mathrm{h}_{\mathrm{s}}-\mathrm{R}_{\mathrm{s}} \mathrm{T}_{\mathrm{RT}}\right)$

where the enthalpies of steam in the input stream and inside the vessel $\left(h_{s}^{i}\right.$ and $h_{s}$ respectively) axe related to the retort temperature by integrating equations of the form: $\frac{d h_{s}}{s T}=C_{p s}(T)$

Integration is performed from a certain reference state to the current temperature. Steam mass in the retort is calculated as:

$$
\begin{aligned}
& m_{s}=\frac{p^{e q} V_{R T}}{R_{s} T_{R}} \\
& p^{e q}=\exp \left(A-\frac{B}{T_{R T}}\right)
\end{aligned}
$$

The heat absorption in the different parts of the retort is resolved into the following terms: 
$\dot{Q}=M_{R T} C_{R T} \dot{T}_{R T}$

$\dot{Q}_{T}=\dot{Q}_{m d}+\dot{Q}_{\text {conv }}+\dot{Q}_{\text {prod }}$

where $\mathrm{Q}_{\mathrm{RT}}$ represents the heat absorbed by the retort body. $\mathrm{Q}_{\mathrm{rad}}$ and $\mathrm{Q}_{\text {conv }}$ represent the heat lost by radiation and convection with the surroundings. The relationships with the retort temperature is:

$\dot{Q}_{\text {rad }}=\theta \varepsilon A_{R T}\left(T_{R T}^{4}-T_{e x t}^{4}\right)$

$\dot{Q}_{\text {conv }}=h_{c} A_{R T}\left(T_{R T}-T_{e x t}\right)$

The heat absorbed by the product, $\mathrm{Q}_{\text {prod, }}$, is estimated by numerically solving the corresponding heat transfer equation with the appropriate boundary conditions (depending on the transfer mechanism and the geometry of the product, as shown in Banga et al., 1993). For the case of conduction-heated type products, the heat transfer equation becomes:

$p C_{\text {prod }} \frac{\partial T_{\text {prod }}}{\partial t}+\nabla\left(\vartheta \nabla T_{\text {prod }}\right)=0$

The overall heat absorbed is then calculated according to the following equations:

$\bar{T}_{\text {prod }}=\frac{\int_{v} T_{\text {prod }}(x, y, z, t) d V}{\int_{v} d V}$

$\dot{Q}_{\text {prod }}=M_{\text {prod }} C_{\text {prod }} \bar{T}_{\text {prod }}$

Substituting the corresponding integrals of (A4) (the reference state is chosen as saturated water at $1 \mathrm{~atm}$ pressure) in (A3), an ordinary differential equation describing the evolution of the retort temperature is obtained. Time derivatives of $\mathrm{m}$, are represented as functions of $\dot{T}_{R T}$ by using the mass balance (A1) and (A5) and (A6). The following relations will be employed in the derivation:

$$
\begin{aligned}
& \dot{m}_{s}=\frac{\partial m_{s}^{e q}}{\partial T_{R T}} \\
& \frac{\partial m_{s}^{e q}}{\partial T_{R T}}=\frac{V_{R T}}{R_{s} T_{R T}^{2}}\left(T_{R T} \frac{\partial p^{e q}}{\partial T_{R T}}-p^{e q}\right) \\
& \frac{\partial p^{e q}}{\partial T_{R T}}=\frac{B}{T_{R T}^{2}} p^{e q}
\end{aligned}
$$


Steam input flow and flow through the bleeder are described by nonlinear equations of the form proposed by Smith and Corripio (1985). As reported by Alonso (1993), these relations resulted in a better agreement than the traditional linear dependence proposed in earlier works (Mulvaney et al., 1990; Alonso et al., 1993)

$\mathrm{F}_{\mathrm{s}}\left(\mathrm{kg} \cdot \mathrm{s}^{-1}\right)=\mathrm{W}_{\mathrm{s}}\left(\mathrm{T}_{\mathrm{RT}}\right) \mathrm{C}_{\mathrm{vs}}$

$\mathrm{F}_{\mathrm{b}}\left(\mathrm{kg} . \mathrm{s}^{-1}\right)=\mathrm{W}_{\mathrm{b}}\left(\mathrm{T}_{\mathrm{RT}}\right) \mathrm{C}_{\mathrm{vb}}$.

For the case of linear valves, the characteristic valve parameters $\mathrm{C}_{\mathrm{vs}}$ and $\mathrm{C}_{\mathrm{vb}}$ are related to valve openings $u_{s}$ and $u_{b}$ by equations of the form (Smith and Corripio, 1985):

$\mathrm{C}_{\mathrm{vs}}=\left(\mathrm{C}_{\mathrm{vs}}\right) \mathrm{u}_{\mathrm{s}}={ }_{1} \mathrm{u}_{\mathrm{s}}$

$\mathrm{C}_{\mathrm{vb}}=\left(\mathrm{C}_{\mathrm{vb}}\right) \mathrm{u}_{\mathrm{b}}={ }_{1} \mathrm{u}_{\mathrm{s}}$

$\mathrm{W}=\left(3.410^{-3}\right) \mathrm{C}_{1} \mathrm{P}_{1}\left(\mathrm{y}_{\mathrm{s}}-0.148 \mathrm{y}_{\mathrm{s}}^{3}\right)\left(\mathrm{C}_{\mathrm{v}}\right)_{\mathrm{u}=1}$

$y_{s}=\frac{1.63}{C_{t}} \sqrt{\frac{P_{1}-P_{2}}{P_{1}}}$

where $\mathrm{P}_{1}$ and $\mathrm{P}_{2}$ are the pressures before and after the corresponding valve (steam valve or bleeder).

\subsection{Derivation of the linear model}

Let us consider a stationary operation point $\left(\bar{T}_{R T}, \bar{u}_{s}, \bar{u}_{b}\right)$ obtained by fixing the positions of steam and bleeder valves and letting $Q_{\text {prod }}=0$. From stationarity, (4) becomes:

$W_{s}\left(\bar{T}_{R T}\right) \bar{u}_{s}=W_{b}\left(\bar{T}_{R T}\right) \bar{u}_{b}+\frac{\dot{Q}_{T}\left(\bar{T}_{R T}\right)}{\Lambda}$

Adding and substracting the term $\mathrm{W}_{\mathrm{s}}\left(\mathrm{T}_{\mathrm{RT}}\right) \mathrm{u}_{\mathrm{s}}$ to the right hand side of (4) and using (A8) we obtain:

$\Omega \Delta \dot{T}_{R T}=\Delta\left(W_{s}\left(T_{R T}\right) u_{s}\right)-\Delta\left(W_{b}\left(T_{R T}\right) u_{b}-\frac{\Delta \dot{Q}_{T}+\dot{Q}_{p r o d}}{\Lambda}\right.$

where $\Delta$ represents deviation with respect to the nominal operation point:

$\Delta\left(\mathrm{W}_{\mathrm{s}}\left(\mathrm{T}_{\mathrm{RT}}\right) \mathrm{u}_{\mathrm{s}}\right)=\mathrm{W}_{\mathrm{s}}\left(\mathrm{T}_{\mathrm{RT}}\right) \mathrm{u}_{\mathrm{s}}-\mathrm{W}_{\mathrm{s}}\left(\mathrm{T}_{\mathrm{RT}}\right) \mathrm{u}_{\mathrm{s}}$

$\Delta\left(\mathrm{W}_{\mathrm{b}}\left(\mathrm{T}_{\mathrm{RT}}\right) \mathrm{u}_{\mathrm{b}}\right)=\mathrm{W}_{\mathrm{b}}\left(\mathrm{T}_{\mathrm{RT}}\right) \mathrm{u}_{\mathrm{b}}-\mathrm{W}_{\mathrm{b}}\left(\mathrm{T}_{\mathrm{RT}}\right) \mathrm{u}_{\mathrm{b}}$

$\Delta \mathrm{Q}_{\mathrm{T}}=\mathrm{Q}\left(\mathrm{T}_{\mathrm{RT}}\right)-\mathrm{Q}_{\mathrm{T}}\left(\mathrm{T}_{\mathrm{RT}}\right)$. 
The terms in (A10) are approximated by its first order Taylor series expansion as follows:

$$
\begin{aligned}
& W_{s}\left(T_{R T}\right) u_{s}-W_{s}\left(\bar{T}_{R T}\right) \bar{u}_{s}=\left(\frac{\partial W_{s}}{\partial T_{R T}}\right)_{T_{R T}} \bar{u}_{s} \Delta T_{R T}+W_{s}\left(\bar{T}_{R T}\right) \Delta u_{s} \\
& W_{b}\left(T_{R T}\right) u_{b}-W_{b}\left(\bar{T}_{R T}\right) \bar{u}_{b}=\left(\frac{\partial W_{b}}{\partial T_{R T}}\right)_{T_{R T}} \bar{u}_{b} \Delta T_{R T}+W_{b}\left(\bar{T}_{R T}\right) \Delta u_{b} \\
& \dot{Q}_{T}\left(T_{R T}\right)-\dot{Q}_{T}\left(\bar{T}_{R T}\right)=\left(\frac{\partial \dot{Q}_{T}}{\partial T_{R T}}\right)_{T_{R T}} \Delta T_{R T}
\end{aligned}
$$

Substituting (A11)(A12)(A13) into (A9) and reordering terms, results an equation of the form of (6) with:

$$
\begin{aligned}
& \tau=\frac{\Omega\left(\bar{T}_{R T}\right)}{f_{\tau}\left(\bar{T}_{R T}, \bar{u}_{s}, \bar{u}_{b}\right)} \\
& K_{s}=\frac{W_{s}\left(\bar{T}_{R T}\right)}{f_{\tau}\left(\bar{T}_{R T}, \bar{u}_{s}, \bar{u}_{b}\right)} \\
& K_{b}=\frac{W_{b}\left(\bar{T}_{R T}\right)}{f_{\tau}\left(\bar{T}_{R T}, \bar{u}_{s}, \bar{u}_{b}\right)} \\
& f_{\tau}\left(\bar{T}_{R T}, \bar{u}_{s}, \bar{u}_{b}\right)=-\left(\frac{\partial W_{s}}{\partial T_{R T}}\right)_{T_{R T}} \bar{u}_{s}+\left(\frac{\partial W_{b}}{\partial T_{R T}}\right)_{T_{R T}} \bar{u}_{b}+\frac{1}{\Lambda}\left(\frac{\partial^{2} Q_{t}}{\partial T_{R T} \partial_{t}}\right)_{T_{R T}} \\
& g\left(\Delta T_{R T}\right)=\frac{\dot{Q}_{p r o d}}{\Lambda f_{\tau}\left(\bar{T}_{R T}, \bar{u}_{s}, \bar{u}_{b}\right)}
\end{aligned}
$$


Fig. 1. Schematic of a batch sterilization steam retort with control equipment.

Fig. 2. Effect of valve positions on the plant dynamics for the case under study. (a) Steam valve gain as a function of valve positions $\left(\mathrm{u}_{\mathrm{s}}\right)$ for different bleeders $\left(\mathrm{u}_{\mathrm{b}}\right)$. (b) Stationary operation temperatures at different valve positions.

Fig. 3. Response of PI controllers with constant and variable parameters. (a) Low temperature tracking. (b) High temperature tracking. In CIMC1, parameters correspond to $\mathrm{T}_{\mathrm{RT}}=130^{\circ} \mathrm{C}, \mathrm{u}_{\mathrm{b}}=0.25$ and $\mathrm{u}_{\mathrm{s}}=0.045$. In CIMC2, parameters correspond to $\mathrm{T}_{\mathrm{RT}}=$ $130^{\circ} \mathrm{C}, \mathrm{u}_{\mathrm{b}}=1$ and $\mathrm{u}_{\mathrm{s}}=0.15$.

Fig. 4. Performance of SAC for a first order model and sampling time of 2 s. (a) Temperature response and valve movements. (b) Parameter evolution.

Fig. 5. Temperature responses obtained with STR for a first order model $\mathrm{K}=0.3$ and a sampling time of 1 s. (a) Temperature response and valve movements. (b) Parameter evolution.

Fig. 6. Block diagram corresponding to the AIMC controller

Fig. 7. Response obtained with AIMC for set point tracking, with $\lambda=20 \mathrm{~s}$ and a sampling time of $1 \mathrm{~s}$. (a) Temperature evolution. (b) Evolution of the nominal gain (MEP) calculated according to (A15) and the corresponding gain estimated recursively (REP). (c) Evolution of the nominal time constant (MEP) calculated according to (A14) and the corresponding time constant estimated recursively (REP).

Fig. 8. Parameter bursting obtained with recursive AIMC for set point tracking, with $\lambda=$ $20 \mathrm{~s}$ and a sampling time of $1 \mathrm{~s}$. (a) Temperature evolution. (b) Evolution of the nominal gain (MEP) calculated according to (A15) and the corresponding gain estimated recursively (REP). (c) Evolution of the nominal time constant (MEP) calculated according to (A14) and the corresponding time constant estimated recursively (REP).

Fig. 9. Response obtained with AIMC for set point tracking with $\lambda=20 \mathrm{~s}$ and a sampling time 10s. (a) Temperature evolution. (b) Evolution of the nominal gain (MEP) 
calculated according to (A15) and the corresponding gain estimated recursively (REP). (c) Evolution of the nominal time constant (MEP) calculated according to (A14) and the corresponding time constant estimated recursively (REP).

Fig. 10. Response obtained with the hybrid AIMC for set point tracking, with $\lambda=20 \mathrm{~s}$ and a sampling time of 1 s. (a) Temperature evolution. (b) Evolution of the "real", nominal (MEP), calculated according to (A15), and the corresponding gain estimated recursively (REP). (c) Evolution of the "real", nominal (MEP), calculated according to (A14), and the corresponding time constant estimated recursively (REP).

Fig. 11. Responses obtained by the hybrid adaptive IMC controller with a second order filter in tracking variable set point profiles. (a) Evolution of temperature in the retort, at the center of the product and movements of the manipulated variable. $\lambda=30 \mathrm{~s}$ and a sampling time of 1 s. (b) Evolution of the estimated gain and time constant.

Fig. 12. (a) Evolution of temperature for the complete sterilization cycle. (b) Evolution of pressure during the cooling stage. (c) Responses of the level control loop: valve opening and mass of water accumulated in the retort 